\title{
ERRATUM
}

\section{"Awake" clipping of cerebral aneurysms: report of initial series}

TO THE READERSHIP: An error appeared in the article by Abdulrauf et al. (Abdulrauf SI, Vuong P, Patel R, et al: "Awake" clipping of cerebral aneurysms: report of initial series. J Neurosurg [epub ahead of print October 21, 2016; DOI: 10.3171/2015.12.JNS152140]).

In Table 1, the number of deaths for the current study was incorrectly reported as 1.5-2.6; the actual mortality was 0 . The corrected table is shown below.

The article has been corrected online as of February 3, 2017.

Saleem I. Abdulrauf, MD

St. Louis University, St. Louis, MO

CORRESPONDING ARTICLE See pp 311-318.

INCLUDE WHEN CITING

Published online February 3, 2017; DOI: 10.3171/2017.1.JNS152140a.

CAANS, 2017

TABLE 1. Contemporary (neurophysiological monitoring era) outcomes from clipping of UIAs

\begin{tabular}{|c|c|c|c|c|c|c|c|}
\hline Authors \& Year & Data Source/Study Design & $\begin{array}{l}\text { No. of } \\
\text { Patients }\end{array}$ & $\begin{array}{l}\text { LOS } \\
\text { (days) }^{*}\end{array}$ & $\begin{array}{l}\text { Discharged } \\
\text { to a Facility } \\
\text { (not home) } \dagger\end{array}$ & $\begin{array}{c}\text { No. of } \\
\text { CVAs } \\
(\%)\end{array}$ & $\begin{array}{c}\text { mRS } \\
\text { Score } \\
\text { (median) }\end{array}$ & Deaths $†$ \\
\hline Barker et al., 2003 & NIS database/retrospective & 3498 & NA & $565(16.1)$ & NA & NA & $73(2.1)$ \\
\hline Barker et al., 2004 & NIS database/retrospective & 3498 & 5 & $565(16.1)$ & $274(7.8)$ & NA & $73(2.1)$ \\
\hline Higashida et al., 2007 & $\begin{array}{l}\text { Health Economics and Outcomes Research Group } \\
\text { of Boston Scientific Database/retrospective }\end{array}$ & 1881 & 7.4 & NA & $249(13.2)$ & NA & 2.50 \\
\hline Alshekhlee et al., 2010 & NIS database/retrospective & 3738 & 4 & NA & $340(9)$ & NA & $60(1.6)$ \\
\hline Hoh et al., 2010 & NIS/retrospective & 4700 & $8.9 \pm 10.1$ & NA & NA & NA & \\
\hline Kotowski et al., 2013 & 60 published studies, meta-analysis/retrospective & 9845 & NA & NA & $692(7.0)$ & NA & $157(1.6)$ \\
\hline McDonald et al., 2013 & Premier Inc. Perspective database/retrospective & 1380 & NA & $232(17)$ & $194(14)$ & NA & $10(0.7)$ \\
\hline Jalbert et al., 2015 & $\begin{array}{l}\text { Medicare provider analysis \& review research } \\
\text { identifiable files/retrospective }\end{array}$ & 4357 & $7.1-9.2$ & $(41.9-45.2)$ & NA & NA & NA \\
\hline Present study & Single center/prospective & 30 & 3 & $1(3)$ & $1(3)$ & 1 & 0 \\
\hline
\end{tabular}

$\mathrm{NA}=$ not applicable.

* Values are median, mean $\pm \mathrm{SD}$, or range.

$\dagger$ Values are number (\%) or percent range. 